\title{
BMMeuroley open Individual vulnerabilities to psychosis after antiepileptic drug administration
}

\author{
Nozomi Akanuma, ${ }^{1}$ Naoto Adachi, ${ }^{2}$ Peter Fenwick, ${ }^{1}$ Masumi Ito, ${ }^{3}$ \\ Mitsutoshi Okazaki, ${ }^{4}$ Koichiro Hara, ${ }^{5}$ Ryouhei Ishii (1) , ${ }^{6}$ Masanori Sekimoto, ${ }^{7}$ \\ Masaaki Kato, ${ }^{7}$ Teiichi Onuma ${ }^{7}$
}

To cite: Akanuma N, Adachi N, Fenwick P, et al. Individual vulnerabilities to psychosis after antiepileptic drug administration.

BMJ Neurology Open 2020;2:e000036. doi:10.1136/ bmjno-2019-000036

Received 22 December 2019 Revised 19 July 2020 Accepted 28 July 2020

\section{Check for updates}

(c) Author(s) (or their employer(s)) 2020. Re-use permitted under CC BY-NC. No commercial re-use. See rights and permissions. Published by BMJ.

${ }^{1}$ South London and Maudsley NHS Foundation Trust, London, UK

${ }^{2}$ Adachi Mental Clinic, Sapporo, Japan

${ }^{3}$ Jozen Clinic, Sapporo, Japan

${ }^{4}$ Department of Psychiatry, National Centre Hospital for Mental, Nervous and Muscular Disorders, NCNP, Kodaira, Japan

${ }^{5}$ Asai Hospital, Togane, Chiba, Japan

${ }^{6}$ Department of Psychiaty, University of Osaka Graduate School of Medicine, Osaka, Japan

${ }^{7}$ Musashino Kokubunji Clinic, Kokubunji, Japan

Correspondence to Dr Naoto Adachi; adacchan@tky2.3web.ne.jp

\section{ABSTRACT}

Background Psychosis often develops after the administration of antiepileptic drugs (AEDs) in patients with epilepsy. However, the individual vulnerability and clinical condition of such patients have been rarely scrutinised. We investigated the effect of individually consistent (trait-dependent) and inconsistent (state-dependent) characteristics.

Methods The individual characteristics, clinical states and psychotic outcome of patients from eight adult epilepsy clinics were retrospectively reviewed over 6 -month periods after a new drug (AED or non-AED) administration between 1981 and 2015.

Results A total of 5018 new drugs (4402 AEDs and 616 non-AEDs) were used in 2015 patients with focal epilepsy. Subsequently, 105 psychotic episodes (81 interictal and 24 postictal) occurred in 89 patients. Twelve patients exhibited multiple episodes after different AED administrations. Traitdependent characteristics (early onset of epilepsy, known presumed causes of epilepsy, lower intellectual function and a family history of psychosis) were significantly associated with the patients who exhibited psychosis. Absence of family history of epilepsy was also associated with psychosis but not significantly. Subsequent logistic regression analysis with a model incorporating family history of psychosis and epilepsy, and intellectual function was the most appropriate $(p=0.000)$. State-dependent characteristics, including previous psychotic history and epilepsy-related variables (longer duration of epilepsy, AED administration, higher seizure frequency and concomitant use of AEDs) were significantly associated with psychotic episodes. Subsequent analysis found that a model including AED administration and previous psychotic history was the most appropriate $(p=0.000)$.

Conclusion Psychosis occurring after new AED administration was related to the individual vulnerability to psychosis and intractability of epilepsy.

\section{INTRODUCTION}

Patients with epilepsy are more likely to develop psychotic symptoms than individuals who do not have epilepsy. ${ }^{1}$ Antiepileptic drugs (AEDs) used for the treatment of seizures can induce psychosis. Psychosis may also be induced by neural damage, repeated seizures, electroencephalography (EEG) discharges, cognitive dysfunction and psychosocial stress. ${ }^{2-4}$ All of the aforementioned variables may have multiple associations with each other, and the inter-relations between them have not been fully investigated. ${ }^{4}$

In general, the epileptic and physical state of a patient is complicated by various factors, including seizure aggravation, adverse effects of other drugs and physical dysfunctions on the administration of a new AED. Thus, the effect of the clinical background should be evaluated simultaneously with the AED administration. Furthermore, approximately 20\%-40\% of psychoses thought to be induced by AED exhibit chronic or recurrent courses after the discontinuation of the causative AED. ${ }^{56}$ These findings suggest that individual vulnerability contributes to development of psychosis, in addition to subsequent seizures and AEDinduced changes. Hence, it is essential to determine whether a given clinical state and individual vulnerability are associated with new AED administration, that is, postantiepileptic drug administration psychosis (PAP).

Herein, we have conducted a comprehensive investigation of the development of PAP during the first 6 months of new drug administration at multiple institutions. AEDrelated factors (eg, class of drugs, dosage and concomitant AED) have been described in a previous study. ${ }^{7}$ In this study, we examined the role of individual vulnerability and clinical background on the development of PAP.

\section{METHODS \\ Definitions}

Psychosis was defined as the presence of hallucinations, delusions or a limited number of severe abnormalities of behaviour, such as gross excitement and overactivity, marked psychomotor retardation, and catatonic behaviour in accordance with the International Classification of Diseases, 10th Revision. ${ }^{8}$ Interictal psychosis was defined as any psychosis occurring in clear consciousness in someone previously diagnosed with epilepsy and not exclusively during or immediately 
following a seizure. ${ }^{9} 10$ Postictal psychosis (PIP) was defined as an episode of psychosis developing within 1 week after a definite seizure or usually a cluster of seizures. ${ }^{1011}$

\section{Participants and instances}

The patients with epilepsy included in this study were registered at the following eight Japanese specialty clinics for adult epilepsy: Adachi Mental Clinic, Asahi Chuo Hospital, Asai Hospital, Jozen Clinic, Musashino Kokubunji Clinic, National Centre of Neurology and Psychiatry, Osaka University Hospital and Tenshi Hospital. Patients were diagnosed with focal (localisation-related) epilepsy in accordance with the conventional international epilepsy criteria. ${ }^{12}$ Patients were treated by consultant neuropsychiatrists qualified in psychiatry and epileptology. The clinical data regarding the administration of new AEDs and non-AEDs (drugs for physical and not neurological or psychiatric disorders) were obtained for the period between 1981 and 2015 and were reviewed comprehensively. The first 6 -month period of treatment with each AED was analysed in patients who were treated with a different class of AED during different periods. Cases (instances) with AED monopharmacy and AED polypharmacy were included. Each case (instance) showed a non-psychotic state (psychosis-free) that lasted for 3 months or longer before the administration of a new drug. Cases (instances) with sufficient records on epilepsy and mental states of 6 months or longer after administration were included in the analysis. The last findings were regarded as the endpoint in interrupted cases (instances) for 1 month or more after administration, that is, discontinuation of the new AED and termination of follow-up. No patient suffered from substance misuse, progressive brain disease, or dementing disorders.

\section{Study items}

\section{Psychotic episodes}

The occurrence of any psychotic episodes (ie, first episode and recurrent) was denoted as PAP during the first 6-month period after new drug administration, which was further subclasified into interictal psychosis (IIP) and PIP. Psychiatric evaluations were performed by eight consultant neuropsychiatrists qualified in psychiatry and epileptology. The inter-rater reliability of psychiatric diagnosis has been described in a previous study. ${ }^{7}$

\section{Trait-dependent (consistent) factors}

The following trait-dependent factors were analysed in this study: (1) sex; (2) family history: presence of a firstdegree relative with epilepsy, psychosis, or both; (3) onset of epilepsy: age at the first afebrile seizure; (4) subtype of focal epilepsy: temporal lobe epilepsy (TLE), frontal lobe epilepsy (FLE), parietal lobe epilepsy (PLE), occipital lobe epilepsy (OLE), localisation-related epilepsy with multiple foci or with undetermined foci (MULE), ${ }^{12}$ which was diagnosed from seizure phenomena, EEG and MRI findings; (5) presumed cause of epilepsy: central nervous system (CNS) infection, head trauma with loss of consciousness, birth complications, CNS anomaly, cerebrovascular diseases and brain tumour; (6) febrile convulsion: episode before the development of epilepsy; (7) lateralisation of abnormality using routine scalp EEG recordings, divided into four categories: left, right, bilateral or none ${ }^{13}$; (8) presence of mesial temporal sclerosis (MTS) on brain MRI according to routine qualitative analysis, categorised into four groups: left, right, bilateral or none ${ }^{14}$; (9) intellectual functioning: normal (full-scale intelligence quotient $(\mathrm{FIQ})>85)$, borderline $(85>\mathrm{FIQ}>70)$, and mildly mentally retarded $(70>\mathrm{FIQ}) \cdot{ }^{15}$

\section{State-dependent (inconsistent) factors}

State-dependent factors analysed in this study were as follows: (1) age at new drug administration; (2) duration of epilepsy: interval between onset and administration; (3) history of psychosis: any psychotic episodes (IIP, PIP or both) antedating administration; (4) type of newly administered drug: AED or non-AED; (5) class of AED and maximum daily dosage; (6) seizure frequency at administration: categorised into six ranks (seizure-free for 3 years or more, less than yearly, yearly, monthly, weekly and daily) ${ }^{13}{ }^{16} ;(7)$ concomitant AED: number of concomitant AED taken at administration; and (8) change in seizure frequency: the difference between the initial and the last seizure frequencies was categorised using a 3-point scale into increased, unchanged or decreased. ${ }^{17}$ Items 6 and 7 were evaluated at three follow-up points: 1 month after (1-4 weeks), 3 months after (5-13 weeks) and 6months after (14-26 weeks).

\section{Statistics}

The trait-dependent factors of patients with and without psychoses after new drug (AED or non-AED) administration during the follow-up periods were compared. The state-dependent factors of cases (instances) with and without psychosis after each new drug administration were compared. Differences among groups were subjected to the analysis of variance for linear variables or the MannWhitney $U$ test for rank-ordered variables. Correlation between categorical variables was examined by contingency table analysis. Subsequently, variables demonstrating significant effects on the development of psychoses on single-variate testing were used for logistic regression analysis with backward stepwise elimination; $p$ values of $<0.05$ were considered statistically significant. All statistical analyses were performed using the Statistical Package for Social Sciences V.14.0.

\section{RESULTS}

\section{Participants and instances}

A total of 2067 patients with localisation-related epilepsy were registered at the eight adult epilepsy clinics, with a mean follow-up duration ranging from 1 to 55 years (mean \pm SD $8.7 \pm 7.7)$. They were administered a total of 5018 new drugs (AEDs and non-AEDs).

\section{Individual consistent variables}

The sample consisted of 1027 men and 1040 women. The age at the onset of epilepsy ranged from 0 to 82 years 


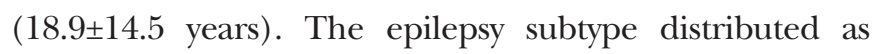
follows: 1109 patients had TLE, 632 had FLE, 62 had PLE, 98 had OLE and 166 had MULE. The presumed cause of epilepsy in 622 patients was as follows: infectious diseases in 133 patients, head traumas in 90, birth complications in 171, anomalies (including cortical dysplasia) in 106, cerebrovascular disorders in 82, brain tumours in 34 and others in 6. The aetiology of epilepsy was unknown in 1445 patients. Moreover, 307 patients had a history of febrile convulsion, while 1708 patients did not have such a history. The lateralisation of EEG abnormalities was as follows: leftsided in 806 patients, right-sided in 820 patients, bilateral in 316 patients and nil in 125 patients. MTS was observed on MRI scans on 248 patients (115 left-sided, 86 right-sided and 44 bilateral). The intellectual function was normal in 1193 and borderline in 462, while 412 patients had mild mental retardation. A family history of epilepsy was observed in 100 patients and was absent in 1967 patients. Forty-one patients had a family history of psychosis, which absent in 2026 patients .

\section{Inconsistent variables at new drug administration}

The age at new drug administration ranged from 12 to 82 years $(35.2 \pm 13.4)$. The duration of epilepsy ranged from 0 to 61 years $(17.8 \pm 12.6)$. The seizure frequency was as follows: less than yearly in 311 , yearly in 1267, monthly in 1569, weekly in 1347 and daily in 397, while 127 cases (instances) were seizure-free. The number of concomitant AEDs ranged from 0 to $6(1.8 \pm 1.1)$.
Of the new drugs administered, 4402 were AEDs (2492 first-generation antiepileptic drug (FAED): 370 phenytoin, 812 carbamazepine, 338 valproate, 258 phenobarbital, 203 clonazepam, 367 clobazam and 146 other FAED; and 1910 second-generation antiepileptic drug (SAED): 351 zonisamide, 496 levetiracetam, 386 gabapentin, 326 topiramate and 349 lamotrigine) and 616 were non-AEDs (222 gastroenterological drugs, 120 cardiovascular/haematological drugs, 117 metabolic/allergic drugs, 113 vitamin supplements and 49 others).

\section{Frequency of psychoses after new drug administration}

The frequency of psychoses has been described elsewhere. ${ }^{7}$ In brief, 105 psychotic episodes (81 IIPs and 24 PIPs) occurred after 5018 new drug administrations in 89 patients. Fifty-five first-episodes of psychosis (47 IIPs and 8 PIPs) occurred after 4658 administrations, and 50 episodes (34 IIPs and 16 PIPs) recurred after 360 administrations. Psychotic episodes were observed once after new drug administrations in 77 patients, two times in eight patients and three times in four patients during the follow-up periods.

\section{Consistent individual variables and psychosis after new drug administration}

A comparison of the consistent individual variables in patients with and without psychosis after new drug administration revealed significant differences in the onset of epilepsy, presumed cause of epilepsy, intellectual function and family history of psychosis (table 1A). Moreover, there

\begin{tabular}{|c|c|c|c|c|}
\hline & $\begin{array}{l}\text { Patients with psychosis } \\
(n=89)\end{array}$ & $\begin{array}{l}\text { Patients without psychosis } \\
(n=1978)\end{array}$ & Statistics & $P$ value \\
\hline Sex (men/women) & $39 / 50$ & $988 / 990$ & $\chi^{2}=1.28^{*}$ & 0.258 \\
\hline Age of onset of epilepsy & 15.5 (SD 11.8) & $19.0(14.6)$ & $F=4.96 \dagger$ & 0.026 \\
\hline Epilepsy subtypes (T/F/P/O/MU) & $45 / 27 / 3 / 4 / 10$ & $1064 / 605 / 59 / 94 / 150$ & $\chi^{2}=1.41^{*}$ & 0.842 \\
\hline $\begin{array}{l}\text { Presumed cause }(\mathrm{Y} / \mathrm{N}) \\
(\mathrm{I} / \mathrm{N} / \mathrm{B} / \mathrm{A} / \mathrm{C} / \mathrm{T} / \mathrm{O} / \mathrm{U})\end{array}$ & $\begin{array}{l}36 / 53 \\
11 / 1 / 9 / 7 / 4 / 3 / 1 / 53\end{array}$ & $\begin{array}{l}586 / 1392 \\
122 / 89 / 162 / 99 / 78 / 31 / 5 / 1392\end{array}$ & $\begin{array}{l}\chi^{2}=4.73^{\star} \\
\chi^{2}=14.4^{\star}\end{array}$ & $\begin{array}{l}0.029 \\
0.044\end{array}$ \\
\hline Febrile convulsion (Y/N) & $15 / 74$ & $298 / 1680$ & $\chi^{2}=0.21^{*}$ & 0.645 \\
\hline $\begin{array}{l}\text { Lateralisation of EEG abnormalities } \\
(\mathrm{L} / \mathrm{R} / \mathrm{B} / \mathrm{N})\end{array}$ & $32 / 38 / 15 / 4$ & $774 / 782 / 301 / 121$ & $\chi^{2}=0.96^{*}$ & 0.812 \\
\hline $\begin{array}{l}\text { MTS on MRI }(\mathrm{Y} / \mathrm{N}) \\
(\mathrm{L} / \mathrm{R} / \mathrm{B} / \mathrm{N})\end{array}$ & $\begin{array}{l}15 / 74 \\
6 / 9 / 0 / 74\end{array}$ & $\begin{array}{l}233 / 1745 \\
111 / 77 / 45 / 1693\end{array}$ & $\begin{array}{l}\chi^{2}=2.08^{*} \\
\chi^{2}=10.4^{*}\end{array}$ & $\begin{array}{l}0.150 \\
0.016\end{array}$ \\
\hline $\begin{array}{l}\text { Intellectual functioning } \\
\text { (N/B/M) }\end{array}$ & $30 / 36 / 23$ & $1163 / 426 / 389$ & $z=-4.09 \ddagger$ & 0.000 \\
\hline Family history of epilepsy (Y/N) & $1 / 88$ & $99 / 1879$ & $\chi^{2}=2.79^{*}$ & 0.095 \\
\hline Family history of psychosis $(\mathrm{Y} / \mathrm{N})$ & $8 / 81$ & $33 / 1945$ & $\chi^{2}=23.5$ & 0.000 \\
\hline
\end{tabular}

Presumed cause: infection (I), neurotrauma $(N)$, birth complication $(B)$, anomaly and migration disorders $(A)$, cerebrovascular $(C)$, tumour $(T)$, others $(\mathrm{O})$, unknown $(\mathrm{U})$; intellectual functioning: normal $(\mathrm{N})$, borderline $(\mathrm{B})$; mildly mentally retarded $(\mathrm{M})$.

Bold type indicates significant $P$ values and statistics $(P<0.05)$.

${ }^{*}$ Contingency table analysis.

†Analysis of variance.

$\ddagger$ Mann-Whitney test.

EEG, electroencephalography; MTS, mesial temporal sclerosis; N, no; Y, yes. 
Table 1B Results of logistic regression analysis with trait-dependent variables

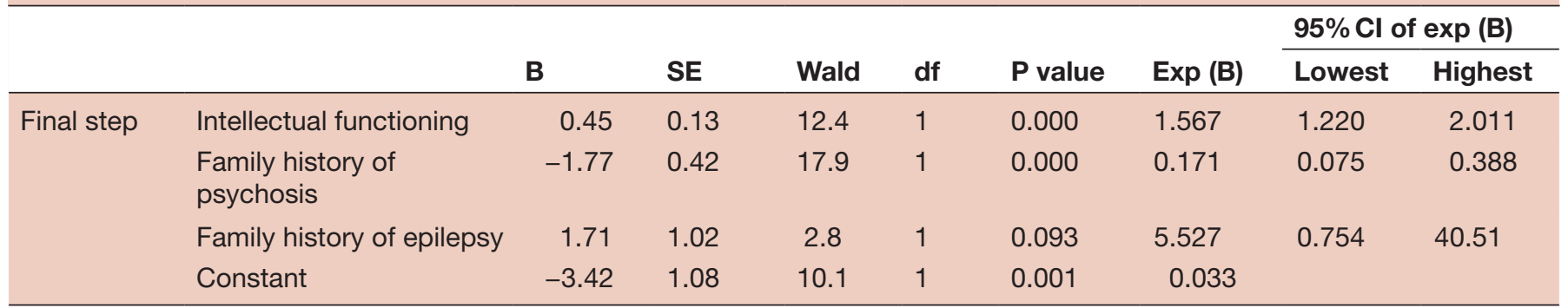

First step: variables entered: age of onset of epilepsy, presumed cause, mesial temporal sclerosis, intellectual functioning, family history of psychosis and family history of epilepsy.

was a significant correlation was observed with MTS if the four categories of MTS were used in the analysis (predominant in right MTS, table 1A). However, no significant correlation was observed if two categories were used, that is, positive or negative MTS (table 1A). There was a slight (insignificant) correlation between a family history of epilepsy and psychosis.

A logistic regression model with backward stepwise procedure was used to determine the actual contribution of these variables involved in the development of psychosis. As shown in table 1B, the model incorporating intellectual function, family history of psychosis and family history of epilepsy was the most appropriate $\left(\chi^{2}=29.8, \mathrm{df}=3, \mathrm{p}=0.000,95.7 \%\right.$ correctly allocated $)$.

\section{Comparison of state-dependent variables between occurrence and non-occurrence of psychosis}

The clinically inconsistent variables at the time of new drug administration are shown in table 2A. Psychosis tended to occur with AED use, longer duration of epilepsy, higher seizure frequency, previous history of psychosis and concomitant use of other AEDs. A detailed description of the effects of each AED on PAP development has been reported elsewhere. ${ }^{7}$ In brief, SAED administration was significantly associated with the development of PAP compared with FAED administration. Among the AEDs, PAP occurred more frequently with topiramate and lamotrigine. Therefore, this study used only the type of new drug administered (ie, AED and non-AED) to clarify background vulnerability.

Logistic regression analysis with a backward stepwise procedure was used to clarify the actual contribution of significant variables to the development of psychosis (table 2B). The model including the type of the new drug administered and history of psychosis showed the highest accuracy $\left(\chi^{2}=144.8, \mathrm{df} 2, \mathrm{p}=0.000,97.9 \%\right.$ correctly allocated).

\section{DISCUSSION}

This study found that individual vulnerability to psychosis and various clinical states were associated with the development of psychoses after new drug administration.

Table 2A Comparison of state-dependent variables between cases with and without psychoses

\begin{tabular}{|c|c|c|c|c|}
\hline & $\begin{array}{l}\text { Psychosis } \\
(n=105)\end{array}$ & $\begin{array}{l}\text { Non-psychosis } \\
(n=4913)\end{array}$ & Statistics & $P$ value \\
\hline Type of drug (AED/non-AED) & $102 / 3$ & $4300 / 613$ & $X^{2}=8.19^{*}$ & 0.004 \\
\hline Age of the new drug administration & 35.8 (SD 12.0) & 35.2 (SD 13.4) & $\mathrm{F}=0.22 \dagger$ & 0.643 \\
\hline Duration of epilepsy & 21.2 (SD 12.6) & $17.7(12.6)$ & $F=8.06 \dagger$ & 0.005 \\
\hline History of psychosis & $50 / 55$ & $310 / 4603$ & $X^{2}=263.4^{*}$ & 0.000 \\
\hline $\begin{array}{l}\text { Seizure frequency } \\
(n /<y / y / m / w / d)\end{array}$ & $2 / 4 / 19 / 34 / 35 / 11$ & 125/307/1248/1535/1312/386 & $Z=-2.40 \ddagger$ & 0.017 \\
\hline Seizure outcome (D/UC/l) & $24 / 73 / 8$ & $1339 / 3350 / 224$ & $Z=-1.39 \ddagger$ & 0.166 \\
\hline Concomitant AED & 2.1 (SD1.1) & 1.8 (SD1.1) & $F=5.73 \dagger$ & 0.017 \\
\hline
\end{tabular}

Seizure frequency: nil $(n)$; less than yearly $(<y)$; yearly $(y)$, monthly $(m)$, weekly $(w)$, daily (d); seizure outcome: decreased (D), unchanged (UC), increased (I).

${ }^{*}$ Contingency table analysis.

†Analysis of variance.

¥Man-Whitney test.

AED, antiepileptic drug. 
Table 2B Results of logistic regression analysis with state-dependent variables

\begin{tabular}{|c|c|c|c|c|c|c|c|c|c|}
\hline & & \multirow[b]{2}{*}{ B } & \multirow[b]{2}{*}{ SE } & \multirow[b]{2}{*}{ Wald } & \multirow[b]{2}{*}{ df } & \multirow[b]{2}{*}{$P$ value } & \multirow[b]{2}{*}{$\operatorname{Exp}(B)$} & \multicolumn{2}{|c|}{$95 \% \mathrm{Cl}$ of $\exp (\mathrm{B})$} \\
\hline & & & & & & & & Lower & Upper \\
\hline \multirow[t]{2}{*}{ Final step } & Type of drug & 1.63 & 0.59 & 7.6 & 1 & 0.006 & 0.196 & 0.062 & 0.627 \\
\hline & Constant & -1.71 & 0.16 & 121.8 & 1 & 0.000 & 0.181 & & \\
\hline
\end{tabular}

First step: five variables administered: duration of epilepsy, type of drug, seizure frequency, concomitant antiepileptic drug and history of psychosis.

These findings are worth considering owing to several advantages, that is, a large placebo-controlled design for multiple AED in a common clinical setting and high reliability of psychiatric evaluations, although they were obtained with retrospective, non-randomised and nonblinded manners. ${ }^{7}$

\section{Trait-dependent (consistent) factors}

The frequency of psychosis after new drug administration was three times higher in patients with a family history of psychosis than that in those without a such history. This observation is strongly suggestive of the significant risk of genetic vulnerability in the development of psychoses, even with PAP. The influence of genetics on PAP remains controversial. ${ }^{18-20}$ The size of these previous study populations appears to be insufficient to consider a low expected frequency of family history. Ever since Slater's seminal study, familial loading of psychosis has long been neglected as a risk factor for psychoses associated with epilepsy. ${ }^{21}$ However, recent large-scale studies have shown the impact of genetic traits on the development of psychoses in general epilepsy cohorts. ${ }^{22}$

Patients with low intellectual function are more liable to exhibit psychosis after new drug administration. Some studies have shown similar tendencies for PAP. ${ }^{20}{ }^{23}$ This finding is consistent with general psychoses with $^{22}{ }^{24}$ or without epilepsy. ${ }^{25}$ In general, individuals with mental and multiple disabilities are prone to adverse events associated with AED use. ${ }^{26}$ Some individuals with mental disabilities may not be able to express unusual experiences after new drug administration and continue taking the medication, allowing for the proliferation of psychosis, ${ }^{27}$ while subtle changes in mental and physical functions may go unnoticed.

Several epilepsy/neural damage-related variables (ie, earlier onset of epilepsy, presumed cause of epilepsy and MTS) were associated with the development of psychoses in this study. Several studies have demonstrated associations between these variables and the development of PAP. $^{20}{ }^{28}$ These variables represent the general risks for the occurrence of psychosis in patients with epilepsy. ${ }^{4} 10$ However, their contribution appears to be limited to an auxiliary role in the process of PAP development after elimination using subsequent multifactorial analysis. Nonetheless, these variables could be used as indicators of potential risks for PAP, especially when other risk variables are unavailable.

\section{State-dependent (inconsistent) variables}

Patients with a previous psychotic history showed a 10-times higher frequency of PAP than those without such a history. Several studies reported similar findings in patients with PAP $^{5} 62829$ and other phenotypes of epilepsy psychoses. ${ }^{10}{ }^{30} \mathrm{~A}$ previous history of psychosis can be an index of the predisposition for psychosis. In particular, $14 \%$ of patients with PAP exhibited multiple psychotic episodes with the administration of different AEDs. In these patients, the vulnerability of such patients to psychosis, irrespective of the class of AED, may play a prominent role in the genesis of PAP.

AED administrations showed a significantly (five times) higher frequency for the development of psychosis than that with non-AED administration. Most non-AEDs used for treating physical illness (which have fewer clinical effects on CNS function) can practically be regarded as control drugs. While psychoses occurred in chronological relation with AED treatment, its contribution to the genesis of psychosis remains uncertain. Only a few controlled studies have focused on the comparison between AEDs and non-AEDs in this regard, except for several clinical trials with placebo-controlled procedures. ${ }^{31-33}$ In the current study, we confirmed the psychogenetic potential of AED. Furthermore, SAEDs have a higher psychogenic potential than that of FAEDs. ${ }^{7}$

Several variables (eg, longer duration of epilepsy, higher frequency of seizures and more concomitant AED) were also associated with the development of PAP. These variables are suggestive of the intractability of epilepsy and an overlap with the requirement of the aforementioned additional AED treatments. We recently reported that the frequency of psychotic episodes increased if the number of previous seizures was higher. ${ }^{16}$ Although these effects were eliminated by subsequent multivariable analysis, they should be considered as certain risk factors for the development of PAP, especially in patients without a previous history of psychosis.

\section{Study limitations}

The limitations of this study must be considered. First, although the study populations (participants who registered comprehensively without arbitrary selection) appear 
to be sufficient, the results may not be fully generalisable to all epilepsy cohorts because patients visiting neuropsychiatry specialist clinics often suffer from various complications (eg, intractable seizures and psychiatric illness). The results can change with subtle deviations in observed frequency for variables of low expected frequency. Second, in this study, cases (instances) with ongoing psychosis at a new AED administration were excluded to facilitate evaluation of the psychogenic effects of AED administration. However, they may have provided valuable information, since these states can be regarded as a lifelong history of psychosis. Third, the study design was as retrospective in nature. Therefore, the reliability of the psychiatric descriptions and evaluations may be limited. However, the high inter-rater correlation of our study suggests sufficient diagnostic reliability of the case descriptions and evaluations. Finally, all episodes of psychoses in our study cannot have been drug-induced, and not all drug-induced psychoses were included. Psychosis induced by other causes (although occurring in response to new drug administration) could be included. In contrast, some cases of drug-induced psychoses could have occurred after the follow-up period. It is difficult to specify a single cause of psychosis in several cases, for example, drug-induced and comorbid schizophrenia. Thus, every psychotic episode applies to the definition analysed.

This study showed that individual vulnerabilities and clinical states were associated with psychosis after AED administration. These findings strongly support our hypothesis that genetic vulnerability and acquired epilepsy-related vulnerability interact to generate psychoses in patients with epilepsy. ${ }^{44}{ }^{35}$ Further explorations of the psychopathological characteristics and effects of each AED are required.

Acknowledgements We thank Edanz Group and Editage (www.Editage.com) for editing a draft of this article.

Contributors NAd, PF and TO conceptualised and designed the study. NAd, MI, MO, $\mathrm{KH}, \mathrm{Rl}, \mathrm{MS}$, MK and TO performed the data collection and data analysis. NAk, NAd, MK, PF and TO interpreted data. NAk, NAd and PF wrote the manuscript. All authors revised and approved the manuscript.

Funding The authors have not declared a specific grant for this research from any funding agency in the public, commercial or not-for-profit sectors.

Competing interests None declared.

Patient consent for publication Not required.

Ethics approval This retrospective study has been approved by local ethics committees (Medical Corporation of Musashi-ya and National Centre of Neurology and Psychiatry, Japan) and has been performed with clinical records of routine treatments and without any further invasive procedures in accordance with the ethical standards laid down in the 1964 Declaration of Helsinki and its later amendments. Patients were informed of their opt-out option if personal identifiable information was being used.

Provenance and peer review Not commissioned; externally peer reviewed.

Data availability statement Data are available upon reasonable request. Data may be obtained from a third party and are not publicly available. Anonymised data not published within this article will be made available by request from any qualified investigators if approved by our institutional ethical committees.

Open access This is an open access article distributed in accordance with the Creative Commons Attribution Non Commercial (CC BY-NC 4.0) license, which permits others to distribute, remix, adapt, build upon this work non-commercially, and license their derivative works on different terms, provided the original work is properly cited, appropriate credit is given, any changes made indicated, and the use is non-commercial. See: http://creativecommons.org/licenses/by-nc/4.0/.

\section{ORCID iD}

Ryouhei Ishii http://orcid.org/0000-0002-4245-0953

\section{REFERENCES}

1 Qin P, Xu H, Laursen TM, et al. Risk for schizophrenia and schizophrenia-like psychosis among patients with epilepsy: population based cohort study. BMJ 2005;331:23-5.

2 Trimble MR. The psychoses of epilepsy. New York: Raven Press, 1991.

3 Schmitz B. Psychiatric syndromes related to antiepileptic drugs. Epilepsia 1999;40:s65-70.

4 Adachi N, Akanuma N. Delusions and hallucinations. In: Mura M, ed. Neuropsychiatry in epilepsy. Springer International Publishing, 2016: 69-89.

5 Matsuura M. Epileptic psychoses and anticonvulsant drug treatment. J Neurol Neurosurg Psychiatry 1999;67:231-3.

6 Kanemoto K, Tsuji T, Kawasaki J. Reexamination of interictal psychoses based on DSM IV psychosis classification and international epilepsy classification. Epilepsia 2001;42:98-103.

7 Adachi N, Fenwick P, Akanuma N, et al. Increased frequency of psychosis after second-generation antiepileptic drug administration in adults with focal epilepsy. Epilepsy Behav 2019;97:138-43.

8 World Health Organization. The ICD-10 Classification of Mental and Behavioural Disorders: Clinical Descriptions and Diagnostic Guidelines. World Health Organization, 1992.

9 Slater E, Roth M. Mayer-Gross, Slater and Roth clinical psychiatry. 3rd edn. Bailliere Tindal, 1969.

10 Adachi N, Kanemoto K, de Toffol B, et al. Basic treatment principles for psychotic disorders in patients with epilepsy. Epilepsia 2013;54:19-33.

11 Logsdail SJ, Toone BK. Post-Ictal psychoses. A clinical and phenomenological description. Br J Psychiatry 1988;152:246-52.

12 Commission on Classification and Terminology of the International League Against Epilepsy. Proposal for revised classification of epilepsies and epileptic syndromes. Epilepsia 1989;30:389-99.

13 Adachi N, Alarcon G, Binnie CD, et al. Predictive value of interictal epileptiform discharges during non-REM sleep on scalp EEG recordings for the lateralization of epileptogenesis. Epilepsia 1998;39:628-32.

14 Adachi N, Kanemoto K, Muramatsu R, et al. Intellectual prognosis of status epilepticus in adult epilepsy patients: analysis with Wechsler adult intelligence Scale-revised. Epilepsia 2005;46:1502-9.

15 American Psychiatric Association. Diagnostic and statistical manual of mental disorders. 4th edn. American Psychiatric Association, 1994.

16 Adachi N, Akanuma N, Fenwick P, et al. Seizure activity and individual vulnerability on first-episode interictal psychosis in epilepsy. Epilepsy Behav 2018;79:234-8.

17 Okazaki M, Adachi N, Akanuma N, et al. Do antipsychotic drugs increase seizure frequency in epilepsy patients? Eur Neuropsychopharmacol 2014;24:1738-44.

18 Mula M, Trimble MR, Lhatoo SD, et al. Topiramate and psychiatric adverse events in patients with epilepsy. Epilepsia 2003;44:659-63.

19 Mula M, Trimble MR, Sander JW. Are psychiatric adverse events of antiepileptic drugs a unique entity? A study on topiramate and levetiracetam. Epilepsia 2007;48:2322-6.

20 Noguchi T, Fukatsu N, Kato $\mathrm{H}$, et al. Impact of antiepileptic drugs on genesis of psychosis. Epilepsy Behav 2012;23:462-5.

21 Slater E, Beard AW, Glithero E. The schizophrenialike psychoses of epilepsy. Br J Psychiatry 1963;109:95-112.

22 Adachi N, Matsuura M, Hara T, et al. Psychoses and epilepsy: are interictal and postictal psychoses distinct clinical entities? Epilepsia 2002;43:1574-82.

23 Chen Z, Lusicic A, O'Brien TJ, et al. Psychotic disorders induced by antiepileptic drugs in people with epilepsy. Brain 2016;139:2668-78.

24 Mellers JD, Toone BK, Lishman WA. A neuropsychological comparison of schizophrenia and schizophrenia-like psychosis of epilepsy. Psychol Med 2000;30:325-35.

25 Aman H, Naeem F, Farooq S, et al. Prevalence of nonaffective psychosis in intellectually disabled clients: systematic review and meta-analysis. Psychiatr Genet 2016;26:145-55.

26 Mattson $\mathrm{RH}$. The role of the old and the new antiepileptic drugs in special populations: mental and multiple handicaps. Epilepsia 1996;37:S45-53. 
27 Kerr M, Scheepers M, Guidelines Working Group, et al. Consensus guidelines into the management of epilepsy in adults with an intellectual disability. J Intellect Disabil Res 2009;53:687-94.

28 Mula M, Monaco F. Antiepileptic drugs and psychopathology of epilepsy: an update. Epileptic Disord 2009;11:001-9.

29 Stephen LJ, Wishart A, Brodie MJ. Psychiatric side effects and antiepileptic drugs: observations from prospective audits. Epilepsy Behav 2017;71:73-8.

30 Onuma T, Adachi N, Hisano T, et al. 10-Year follow-up study of epilepsy with psychosis. Jpn J Psychiatry Neurol 1991;45:360-1.

31 Ferrie CD, Robinson RO, Panayiotopoulos CP. Psychotic and severe behavioural reactions with vigabatrin: a review. Acta Neurol Scand 1996;93:1-8.
32 Levinson DF, Devinsky O. Psychiatric adverse events during vigabatrin therapy. Neurology 1999;53:1503-11.

33 Sackellares JC, Krauss G, Sommerville KW, et al. Occurrence of psychosis in patients with epilepsy randomized to tiagabine or placebo treatment. Epilepsia 2002;43:394-8.

34 Adachi N, Akanuma N, Ito M, et al. Epileptic, organic and genetic vulnerabilities for timing of the development of interictal psychosis. Br J Psychiatry 2010;196:212-6.

35 Adachi N, Akanuma N, Fenwick P, et al. Seizure frequency at the time of first-episode interictal psychosis. Epilepsy Behav 2018;79:234-8. 